\title{
Application of Unmanned Aircraft Systems for Coastal Mapping and Resiliency
}

Michael J. Starek

Conrad Blucher Institute for Surveying and Science, Texas A\&M UniversityCorpus Christi, USA

\section{Melanie Gingras}

Conrad Blucher Institute for Surveying and Science, Texas A\&M UniversityCorpus Christi, USA

\section{Gary Jeffress}

Conrad Blucher Institute for Surveying and Science, Texas A\&M UniversityCorpus Christi, USA

This chapter provides an overview of UAS technology with focus on surveying and mapping. A case study on the use of UAS for coastal monitoring to aid community resiliency following a hurricane impact is also presented. The information and applications of UAS presented herein are applicable to a variety of UN SDGs including sustainable land use for "Life on Land" and sustainable agriculture for crop security and "Zero Hunger".

\subsection{Introduction}

Coastal zones are some of the most dynamic environments on Earth and some of the most threatened. According to the United Nations (UN) Atlas of the Oceans, $44 \%$ of the world's population (more people than inhabited the entire globe in 1950) live within 150 kilometers of the coast [2]. Growing population demand, impact from storms, climate change, and relative sea level rise puts coastal communities at the forefront of engineering and scientific efforts for sustainable and resilient development. As part of the UN Sustainable Development Goals (SDGs) for 2030, Goal 11 specifically identifies "Sustainable 
Cities and Communities". In the context of coastal communities, resilience is a measure of the extent to which a coast is able to respond to external pressures without losing actual or potential functions [7]. Improving coastal resilience is considered to be a cost-effective approach to prepare for increasingly uncertain coastal environments. The ability to rebound more quickly can reduce negative human health, environmental, and economic impacts [5].

At a base level in building more resilient communities is the need for updated geospatial information. Coastal communities rely on adequate and timely geospatial data to guide decision-making in the event of a disaster, mitigate coastal erosion, and plan for sustainable development and growth. Emerging technology for the acquisition of spatially referenced data are rapidly transforming science, society, and decision-making. At the forefront of this revolution are technological advancements in unmanned aircraft systems (UAS), more commonly referred to as drones. UAS enable us to rapidly map and monitor our evolving world, with unprecedented detail, to tackle a range of problems in support of UN SDGs.

\subsection{Overview of UAS Technology}

UAS provide a new paradigm for aerial surveying and mapping. UAS are used to collect overlapping imagery, which can then be post-processed to derive two-dimensional (2D) and three-dimensional (3D) mapping products for geographic information. These data products can be used to characterize built and natural environments at a level of spatial detail previously unattainable or not practical with traditional remote sensing techniques. Spectral data can also be acquired from multispectral sensors onboard the UAS for performing traditional remote sensing tasks such as mapping vegetation health. Compared to traditional aircraft or satellite remote sensing, UAS provides certain advantages: rapid deploy capabilities, flexibility to target ideal weather conditions and for temporal repeatability, hyperspatial image resolutions, and cost-effectiveness at localized geographic extent [10, 12].

Application of UAS for surveying and mapping is primarily conducted with small UAS equipped with consumer-grade digital cameras and miniaturized sensors. Currently, the Federal Aviation Administration (FAA) of the United States classifies small UAS as weighing less than $25 \mathrm{~kg}$ (55 lbs) including payload. Often these platforms weigh only a few kgs with payload. UAS can be broadly classified into two types: rotary craft and fixed-wing (Figure 7.1).

Rotary craft typically provide more flexibility in sensor payload integration, enable more stabilized flight in windy conditions, provide vertical take-off and landing (VTOL) capability, and are better suited for inspection surveying (e.g. hovering), videography, and flying lower or slower to collect higher resolution imagery with reduced motion blur. In contrast, fixed-wing UAS 


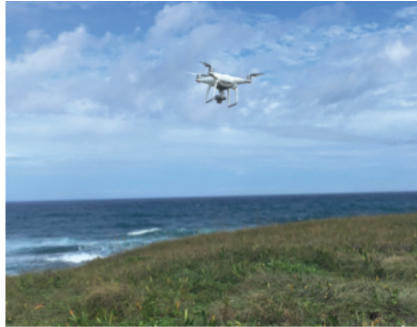

a.

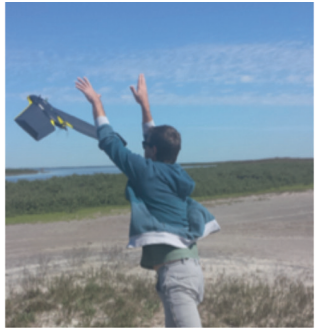

b.

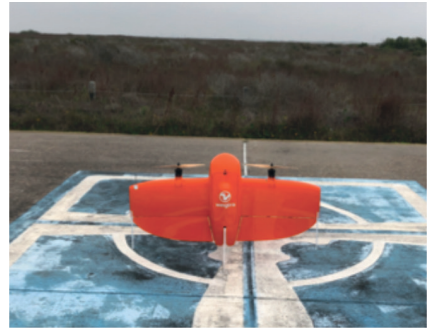

C.

\section{FIGURE 7.1}

Examples of different types of small UAS mapping platforms operated by of the Conrad Blucher Institute for Surveying and Science at Texas A\&M University-Corpus Christi. (a) Rotary platform called the DJI Phantom 4 Pro; (b) Fixed-wing UAS called the SenseFly eBee being hand-launched by the author; (c) Hybrid UAS called the Wingtra that is a vertical take-off and landing (VTOL) system which converts to fixed-wing during flight.

are generally more efficient at mapping larger areas due to more effective lift generation for a given payload thereby maximizing battery endurance $[4,9]$. The majority of current hobbyist UAS activity is centered around small rotary platforms, such as the DJI Phantom series (see Figure 1a), due to their widespread availability, ease of use, and lower cost compared to commercial fixed-wing platforms designed for mapping. There are also hybrid platforms integrating rotary and fixed-wing design that provide the advantages of both, such as a vertical take-off and landing with fixed-wing flight (Figure 1c). With continued technological progression, the endurance and efficiency of UAS will improve and differences in performance capabilities between platform types will become more blurred.

Main system components of a UAS include the global navigation satellite system (GNSS) receiver for position, inertial measurement unit (IMU) for orientation, radio link for communication, onboard processor, and sensor/camera for data acquisition. Autonomous flight is performed using an autopilot that is fed by the onboard GNSS, IMU, and other sensors. UAS often include other navigation aiding sensors, such as magnetometers and pivot tubes, as well as sense-and-avoidance features with built-in artificial intelligence to reduce risk of collision. Sensors equipped on UAS can range from visual RGB digital cameras to multispectral and hyperspectral sensors, thermal imagers, and active sensors such as light detection and ranging (lidar). UAS appropriate for mapping include easy-to-use onboard autopilots for controlling image acquisition, which is important for image stitching and photogrammetric applications. The onboard radio is also essential because it dictates the strength and length of the command and control link between the operator's Ground Control Station (GCS or remote controller; typically, a laptop, tablet, or smart phone 
with mission control software for automated flight) and the UAS, which has implications for both data collection and safety.

\subsection{Aerial Mapping with UAS}

Two-dimensional image mosaics are the most commonly created products from imagery collected by a UAS. The simplest way to create a mosaic from aerial imagery is by using photo stitching software, which combines a series of overlapping aerial photographs into a single image called an image mosaic. However, this approach does not account for perspective distortion and camera lens distortion thereby resulting in non-uniform scale and inaccurate distance measurements for geospatial applications [6]. An orthomosaic is a mosaicked series of overlapping aerial images that have been geometrically corrected (orthorectified) using an elevation model of the terrain to give them a uniform scale. Orthorectification removes distortion from the images by adjusting for topographic relief, lens distortion, and camera tilt creating an aerial map. Orthormosaics are a fundamental geographic information system (GIS) data product used in a variety of planar mapping applications.

Another commonly created geospatial data product from UAS imagery is a digital surface model (DSM). A DSM provides a "2.5D" digital representation of the three-dimensional landcover and exposed ground. It is created from a dense set of georeferenced 3D point measurements called a point cloud derived from the overlapping UAS imagery using specialized photogrammetric processing. The DSM is a raster data type that can be easily ingested into a GIS for further analysis such as to measure the height of an object or estimate the volume of an object. If the data is tied to a vertical datum, a DSM can be considered a specialized type of digital elevation model (DEM) where raster cell values represent the elevation of the exposed ground and landcover. By generating DSMs from repeat UAS surveys across time, the change in elevation can be measured by raster differencing. For example, along a sandy beach, repeat UAS surveys can be used to generate DSMs and then differenced to measure change in elevation and estimate volumetric sediment loss or gain. The generated DSM can also be used by photogrammetric software to orthorectify the UAS imagery and create the 2D orthomosaic product described above. Furthermore, through automated ground point filtering, a bare-earth DEM (called a digital terrain model or DTM) can be created from the set of points identified as stemming from the ground. The accuracy and success of such a process depends highly on the density of landcover and the method implemented by the software. The sections below outline UAS mission planning, flight design, and photogrammetric processing to derive 2D and 3D mapping products. 


\subsubsection{Mission Planning}

Planning a mapping mission with a small UAS entails a number of considerations. A first-order decision to be made is whether the flight will be conducted under autonomous control with mission planning and flight control software using GNSS waypoints or conducted manually. The decision of whether to use manual or autonomous control hinges on your application. As a general rule, autonomous control is more useful when one is trying to fly in a systematic pattern to acquire imagery for aerial mapping and photogrammetry purposes. In contrast, manual control is generally used for events that require reacting to information in real time such as inspection surveying (e.g. wind turbine) or video monitoring and streaming of events in real time (e.g. search and rescue operations). Both types of missions can be flown in either manner, or in a hybrid of manual and automatic control [6].

Prior to conducting any UAS flight operation it is important to analyze the area to be mapped before liftoff. The area should be visually inspected by walking or driving the premises or otherwise evaluated before the mission starts so as to identify obstacles such as power lines, water bodies, large trees, sensitive areas, or other potential pitfalls. It is good practice to use existing aerial or satellite imagery to inspect the area and plot out a flight before takeoff. Pilots should know how to competently fly their UAS, even if they plan to use it primarily for autonomous mapping missions. This means understanding the launch and recovery procedure, flight control software, endurance limitations, trouble shooting, and safety procedures. UAS should remain within the visual line of sight of pilots and ground observers unless the pilots have special regulatory permission by their respective regulatory agency for beyond visual line of sight (VLOS) operation.

\subsubsection{Flight Design}

Designing a flight plan for autonomous mapping is done using mission planning and control software (Figure 7.2).

These are often proprietary software provided by the UAS manufacturers; however, there are open-source solutions. Mission Planner is a current opensource software package widely used by the hobbyist community that works with various compatible autopilots. There are also numerous "flight apps" designed to work off of smart phones and tablets for programming flight missions. The functionality for most of this software, regardless of modality, is generally similar. UAS aerial mapping missions are usually flown in a specific pattern of parallel lines, commonly described as transects, which are connected by a series of geopositional waypoints [6]. These flight patterns are a method of ensuring that the UAS captures an adequate quantity of images at the desired amount of image overlap. Overlap is essential for high fidelity photogrammetric processing to derive mapping products and described in more detail below. Once the mission plan is completed to satisfaction, it is uploaded to the UAS 
controller and the system is ready for launch. The operator can then launch the drone using the manufacturer's prescribed launch procedure.

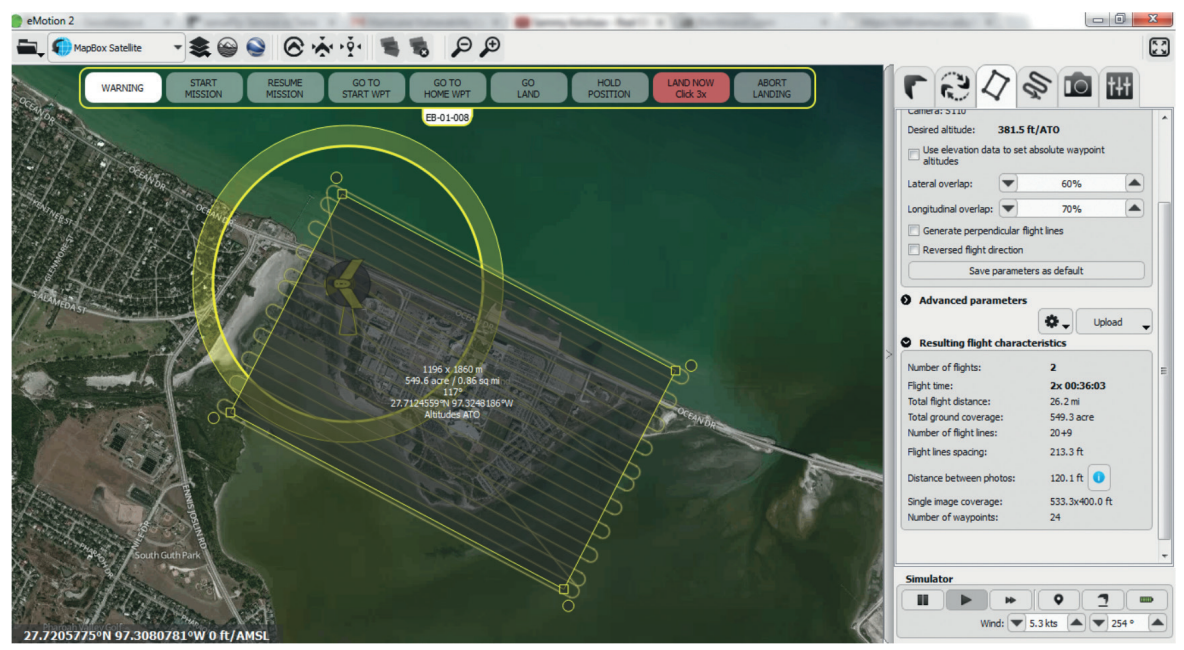

\section{FIGURE 7.2}

Example of a UAS flight design created with proprietary mission planning software called eMotion 2 by SenseFly. Parallel transects can be scene along with image overlap settings. The area shown is the Texas A\&M UniversityCorpus Christi island campus.

Imagery collected during flight is typically stored onboard the platform using a SD memory card located in the camera or via other data storage means. There are services that aim to provide real-time wireless transfer of collected imagery to a cloud architecture during flight for downstream rapid processing using a cellular network or other wireless connection mode. Similar approaches can be implemented for wireless transfer of image data from platform to ground control station. But feasibility of these approaches will depend on bandwidth, available wireless connectivity, size and amount of imagery, and other factors. Presently, the most common operating mode for image acquisition is onboard storage and download of imagery after landing.

\subsubsection{Image GSD and Overlap}

To successfully perform UAS mapping, proper flight design is critical. Two important parameters to consider in designing any UAS survey are ground sample distance (GSD) and image overlap. GSD is the projected pixel width on the ground and is a function of the camera focal length, physical size of the individual sensor elements inside the camera (called pixel pitch), and flying height above ground level. Figure 7.3 shows this relationship.

GSD can be estimated as follows: 


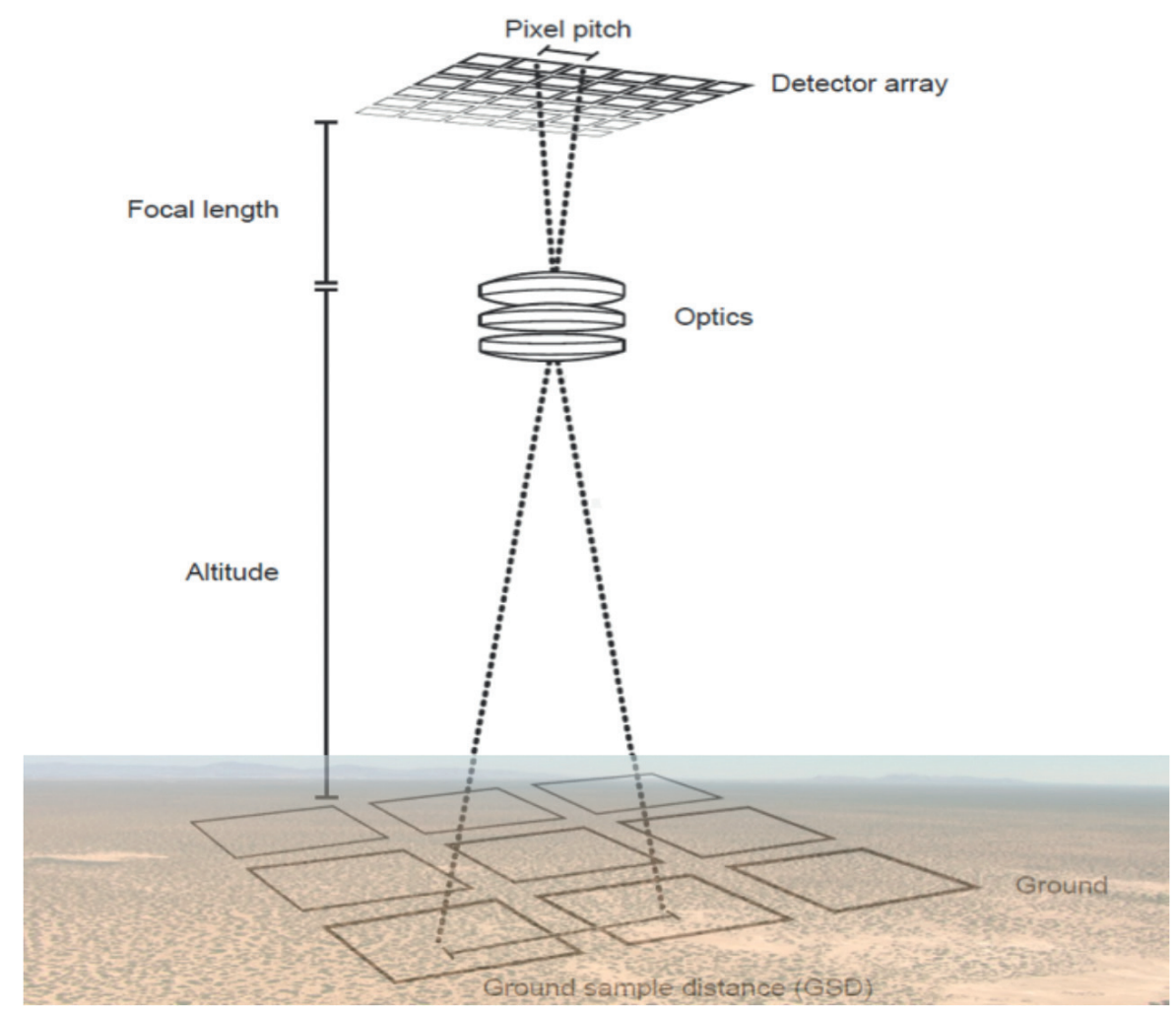

\section{FIGURE 7.3}

Relationship between ground sample distance (GSD), flying height above ground, camera pixel pitch and focal length. (Modified from [6]).

$$
G S D=\frac{(p)(h)}{f}
$$

where $f=$ focal length of the respective camera, $h=$ flying height above ground level, $p=$ pixel pitch. During flight design, mission planning software will allow one to specify the specific camera model and focal length or it will be autodetected by the software. The software then allows one to adjust the nominal flying height above ground level to achieve a desired GSD for a given camera model. Dependent on project GSD requirements, the flight altitude may need to be adjusted during the mission to compensate for large variation in terrain elevation. Certain UAS can be programmed to use elevation models to follow the terrain for image acquisition.

The second primary consideration for flight design is image overlap. Sufficient overlap is crucial due to the type of photogrammetry employed to process the UAS imagery and reconstruct a 3D model of the imaged scene. In a typi- 
cal scenario, images are acquired with at least $75 \%$ frontal overlap along the flight line and at least $60 \%$ side overlap between adjacent flight lines. For most scenarios, it is recommended that images are acquired with a regular grid pattern (Figure 7.4).

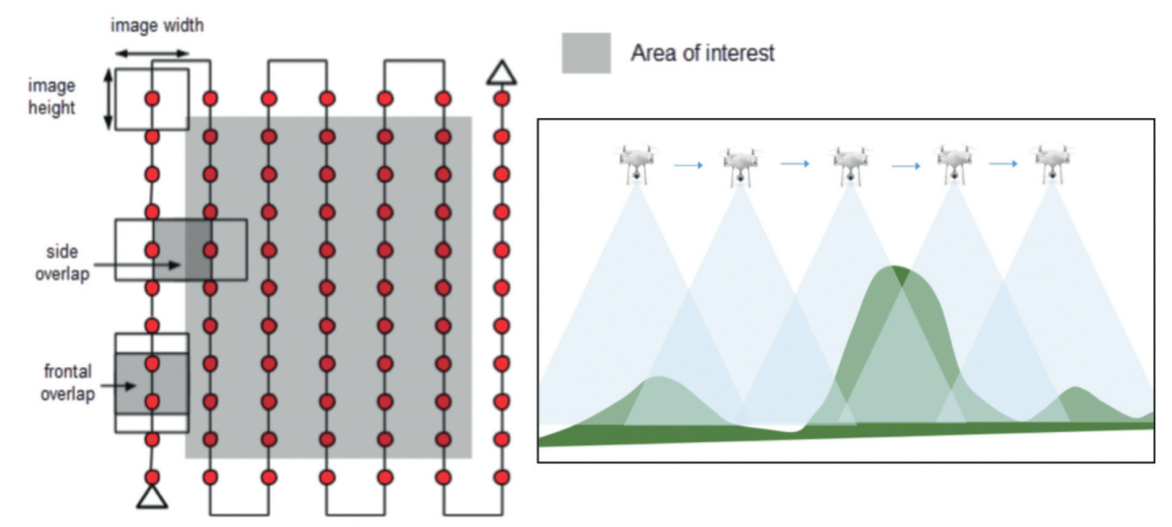

\section{FIGURE 7.4}

Grid image acquisition plan showing the meaning of frontal overlap and side overlap (Source of grid image: [1]). Inlay shows along track frontal overlap.

Over more complex environments, more overlap and different flight patterns may be necessary to achieve desired mapping results. For example, terrain covered by trees and dense vegetation or flat terrain with homogeneous texture pose challenges for photogrammetry software to auto-identify characteristic points (keypoints) shared between overlapping images. This correspondence is necessary for 3D reconstruction from overlapping images. Such cases may require more overlap in both directions and fling higher can sometimes improve results. For details on flight design for UAS mapping, the reader is referred to [1] and [3].

\subsubsection{Structure-from-Motion Photogrammetry}

Photogrammetry is the science of making measurements of objects from photographs. Common survey products include 2D orthorectified image mosaics and 3D models of the imaged scene. Traditional airborne photogrammetry utilizes large-format metric cameras precisely calibrated such that their interior properties, like focal length, are accurately known. However, metric cameras are expensive and not conducive for widespread use of UAS for mapping applications. In contrast, small UAS mapping typically employs consumergrade digital cameras using a technique called Structure-from-Motion (SfM) photogrammetry. SfM exploits information from multiple overlapping images to extract 3D object information and perform self-calibration of the camera negating the need for precisely calibrated metric cameras. SfM derives 
three-dimensional structure from two-dimensional image sequences through movement of the camera thereby providing different perspective views of the scene. By using the UAS as the moving platform, SfM can be implemented with an onboard camera by acquiring images with sufficient overlap as previously explained. The SfM image processing workflow is summarized as follows [8]:

1. Image sequences are input into the software and a keypoint detection algorithm, such as the scale invariant feature transform (SIFT), is used to automatically extract features and find keypoint correspondences between overlapping images using a keypoint descriptor.

2. A "bundle block" adjustment is performed to minimize the errors in the correspondences by simultaneously solving for camera interior and exterior orientation. Based on this reconstruction, the matching points are verified and their 3D coordinates calculated to generate a sparse point cloud. Without any additional information, the coordinate system is arbitrary in translation and rotation and has inaccurate scale.

3. To further constrain the problem, ground control points (GCPs) can be used to transform the point coordinates to a real-world coordinate system and improve rectification. Similarly, initial camera positions from the onboard GNSS can also be used to constrain and georeference the solution.

4. Finally, the interior and exterior orientation for each image are used as input into a Multi-View Stereo (MVS) algorithm, which attempts to densify the point cloud by projecting every image pixel, or at a reduced scale.

The base data product output from UAS-SfM processing is a densified 3D point cloud of the imaged scene colored by the RGB values of the camera. UAS-SfM point clouds can be considered hyperspatial (densely-spaced points exceeding $1000 \mathrm{pts} / \mathrm{m} 2$ ) due to the high camera resolution (e.g. $20 \mathrm{MP}+$ ) and typical low altitudes at which data are collected (Figure 7.5).

The 3D point cloud can then be used by software to create a DSM of the imaged scene, which can subsequently be used to correct distortion in the imagery and generate a seamless orthomosaic. As a result, geospatial data products typically output from a UAS-SfM survey are the following: 3D point cloud, DSM, orthomosaic. Several commercial and open-source SfM software options are available to process UAS imagery. Widely used commercial software include Pix4D and Agisoft PhotoScan. Open Drone Map is a popular open source solution. Figure 7.6 summarizes the UAS-SfM processing workflow.

UAS are integrated with onboard GNSS receivers that can be used to geotag the acquired imagery with respective latitude and longitude coordinates thereby allowing them to be positioned on earth (georeferencing). However, the quality of GNSS receivers onboard these platforms can highly vary. Most 

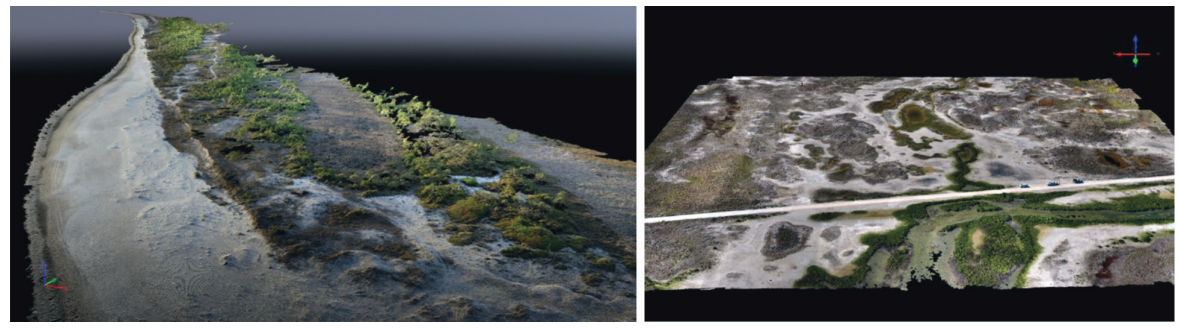

\section{FIGURE 7.5}

(left) RGB colored 3D point cloud of Little St. George Island, FL USA. (right) RGB colored 3D point cloud of a wetland study site located on Mustang Island, TX USA. These point clouds were generated from UAS imagery using structure-from-motion photogrammetry. They are very dense with $>1000$ $p t s / m^{2}$

small UAS come equipped with low-accuracy GNSS receivers used for navigation only that provide 3D positional accuracies on the order of several to tens of meters whereas some systems come equipped with differential GNSS receivers capable of delivering horizontal and vertical positional accuracies down to a few centimeters. To improve positional accuracy of derived mapping products, ground control targets can be uniformly distributed throughout the study site and their positional coordinates accurately surveyed using a traditional land surveying approach, such as real-time kinematic (RTK) GNSS. These ground control points can then be input into the SfM processing workflow to more accurately locate the derived geospatial data products.

\subsection{Regulations}

Any UAS flight conducted for commercial or hobbyist use must ensure that it adheres to appropriate airspace regulations and that proper safety precautions are taken. For example, in the United States, commercial use of UAS for aerial surveying or other applications is regulated by the FAA's small UAS rule (called Part 107), that became effective on August 29, 2016. Current highlights of the operational rules include: aircraft must weigh less than 25 $\mathrm{kg}$ ( 55 pounds) and be registered with the FAA; operation is limited to Class $\mathrm{G}$ airspace, within visual line-of-sight, under 400 feet, daylight hours, and at or below 161 kilometers per hour. Part 107 provides an option to apply for a certificate of waiver, which allows for a small UAS operation to deviate from certain operating rules if the FAA finds that the proposed operation can be performed safely.

Finally, it is important to mention that at the time of this writing (2019), 

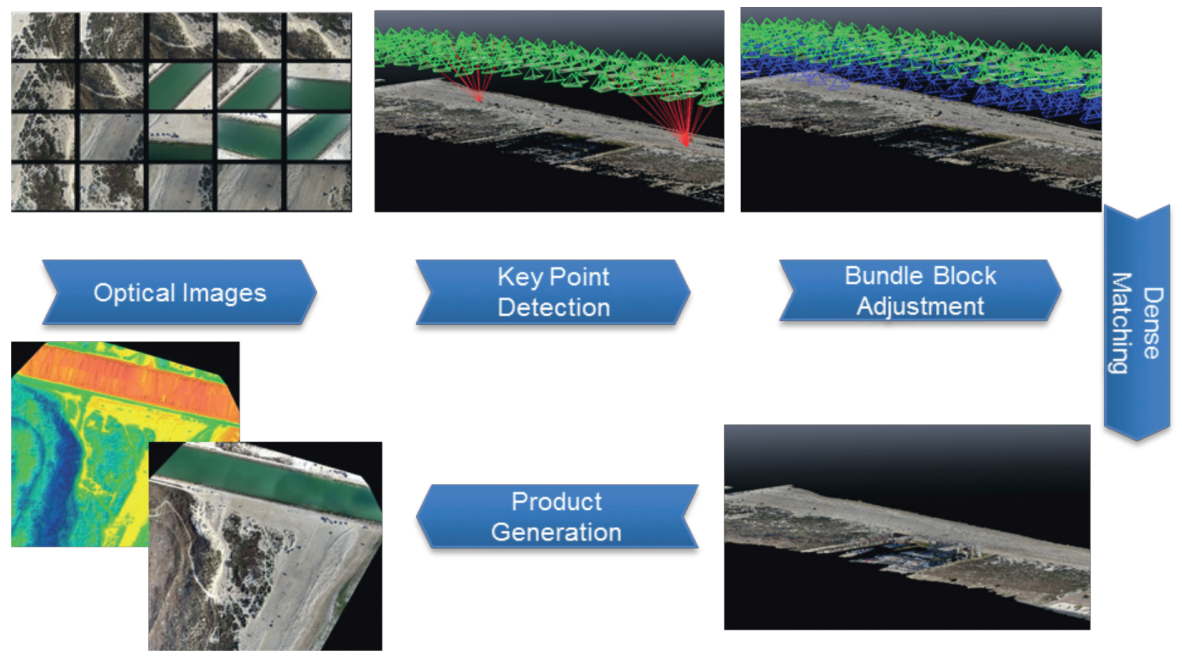

\section{FIGURE 7.6}

SfM workflow to process UAS image sequences into densified 3D point cloud, DSM, and orthomosaic. Example here is from Packery Channel; an open-water inlet located along the Texas Gulf coast

UAS technology and regulations are still emerging. The technology, regulations, and capabilities for surveying will rapidly evolve over the coming years. However, the sensor types, data products and general methods for data acquisition and processing, as discussed here, will likely remain similar.

\subsection{Case Study: Hurricane Harvey Impact Assessment}

Hurricane Harvey formed as a tropical storm over the Atlantic Ocean on August 17, 2017, weakened to a tropical depression as it crossed the Yucatan Peninsula, but rapidly intensified to a Category 4 strength hurricane in the Gulf of Mexico just before making landfall on the Texas coast 50km east of Corpus Christi on August 25. Hurricane Harvey caused severe wind damage in coastal towns, extensive flooding as it stalled over Texas from August 25-30, 2017, and unprecedented rainfall with totals in Cedar Bayou reaching 1318 $\mathrm{mm}\left(58.59^{\prime \prime}\right)$ in just three days [11].

Shortly after the hurricane, the Measurement Analytics (MANTIS) Lab with the Conrad Blucher Institute for Surveying and Science at Texas A\&M University-Corpus Christi conducted small UAS surveys at various beach locations along Mustang Island, TX to assess coastal erosion. Study sites investigated included Gulf-facing beach sites at the Port Aransas jetty, which was 
exposed to the southern edge of the eyewall, and Newport Pass located about $25 \mathrm{~km}$ to the south (Figure 7.7).

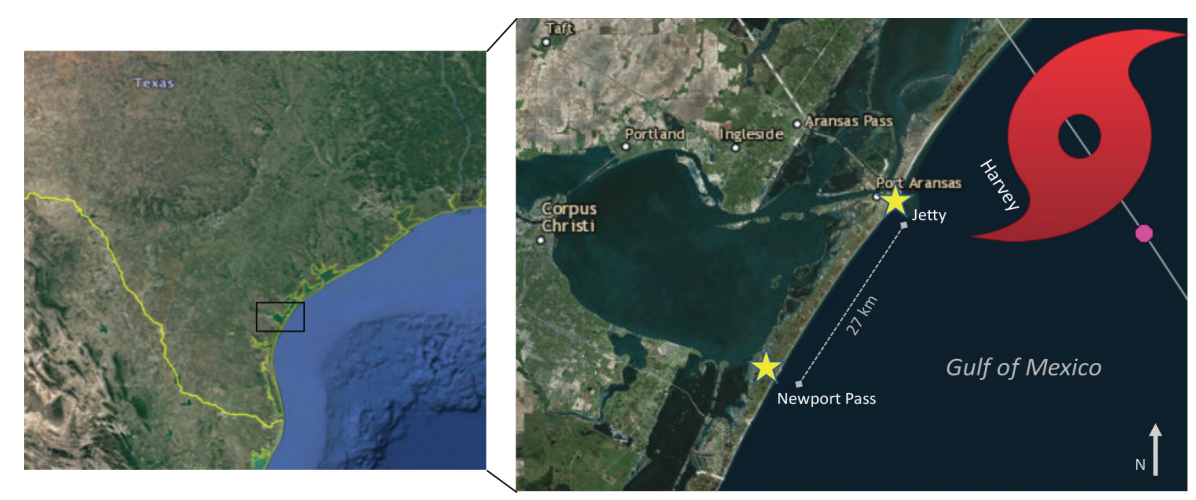

\section{FIGURE 7.7}

Hurricane Harvey Mustang Island, TX study sites

Change at the beach locations was distinguished by comparing posthurricane UAS survey data with pre-storm airborne lidar data collected by the United States Army Corps of Engineers (USACE) National Coastal Mapping Program. The USACE airborne lidar survey was conducted in August-October 2016. The MANTIS lab collected post-impact UAS data in September-October 2017. Newport Pass study site was surveyed using a rotary DJI Phantom 4 Pro (Figure 7.1a), and Port Aransas study site was surveyed using a fixed-wing SenseFly eBee. Both systems were integrated with $20 \mathrm{MP}$ RGB cameras. Ground control targets were used to ensure high-accuracy georeferencing and to tie the data to a vertical datum. All UAS imagery were processed using SfM to generate orthomosaics and DSMs. Because the UAS DSM raster cell values were referenced to a vertical datum for elevation, they represent elevation of the exposed ground and landcover and are referred to herein as DEMs.

Figure 7.8a is a traditional aerial image acquired prior to the hurricane at the Port Aransas South Jetty site, and Figure 7.8b shows a high resolution (3 $\mathrm{cm}$ ) UAS orthomosaic produced from a survey a few weeks after the hurricane.

Results show that the most noticeable change was the beach erosion and scouring near the jetty and along the roadway where as much as $4 \mathrm{~m}$ was devoured by the large wave action and storm surge. The UAS-derived mapping products provided up-to-date information to the County to aid damage assessment of the jetty and guide their recovery efforts. At Newport Pass, there was perhaps the most noticeable change where a previously weakened dune blowout (Figures 7.9a and c) was completely breached by the storm surge during the hurricane and a temporary inlet to Corpus Christi Bay was formed (Figures $7.9 \mathrm{~b}$ and d). This resulted in elevation changes upwards of $4 \mathrm{~m}$ losses where the channel cut through the dune, and gains where the flooded bay wa- 


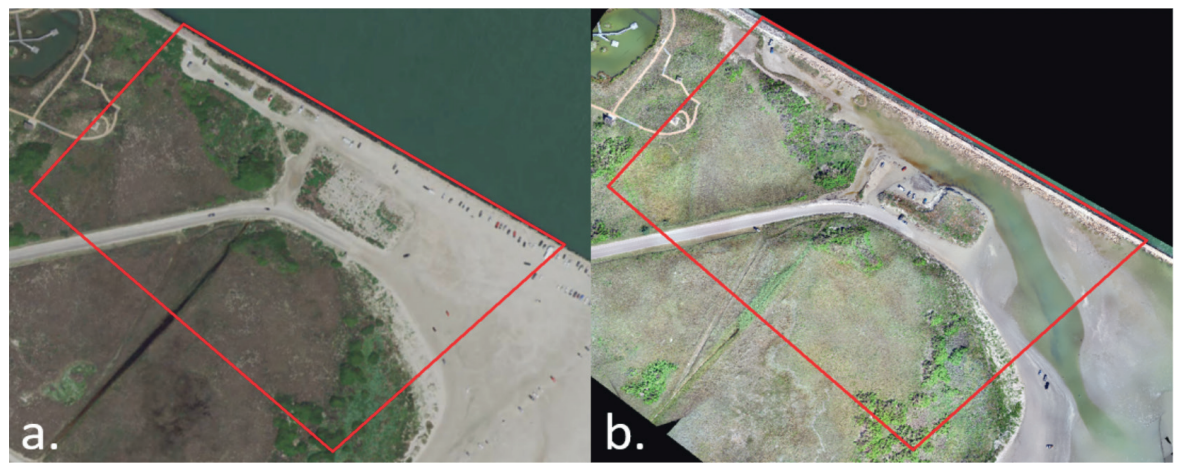

\section{FIGURE 7.8}

These images depict storm-related changes to the Port Aransas South Jetty site. (a) Google Earth aerial image from south of the Aransas Pass jetty before Hurricane Harvey; (b) High resolution UAS imagery from south of the Aransas Pass jetty after Hurricane Harvey.

ters and strong northwest winds from the southern eyewall pushed sediment and water against the backside (bayside) of the dune (Figure 7.9e).

In support of a different initiative related to Harvey, UAS surveys were conducted over a heavily damaged inland neighborhood near Rockport, TX where some of the strongest wind fields were observed during the storm. The effort was conducted as part of a volunteer emergency response effort in collaboration with engineers from the University of Notre Dame and the United States National Science Foundation (NSF) Geotechnical Extreme Events Reconnaissance (GEER) project. The purpose of the NSF GEER effort is to collect high-resolution remote sensing data along with information on structural damage recorded by reconnaissance teams on the ground to evaluate performance of structures during disaster events. Figures 7.10a and 7.10b show aerial images of the neighborhood prior to Hurricane Harvey and Figures 7.10c and 7.10d show parts of a UAS orthomosaic of the same area generated after the storm. Figure 7.10e is an oblique UAS image of six houses before the storm and Figure $7.10 \mathrm{f}$ is an oblique image of a 3D point cloud constructed from the UAS imagery using SfM. The collected UAS information from Harvey and other storms are being used by engineers to improve structural design and refine building codes for reducing impacts from hurricanes and other natural disasters. This effort will lead to more sustainable development and in return, more resilient coasts. 

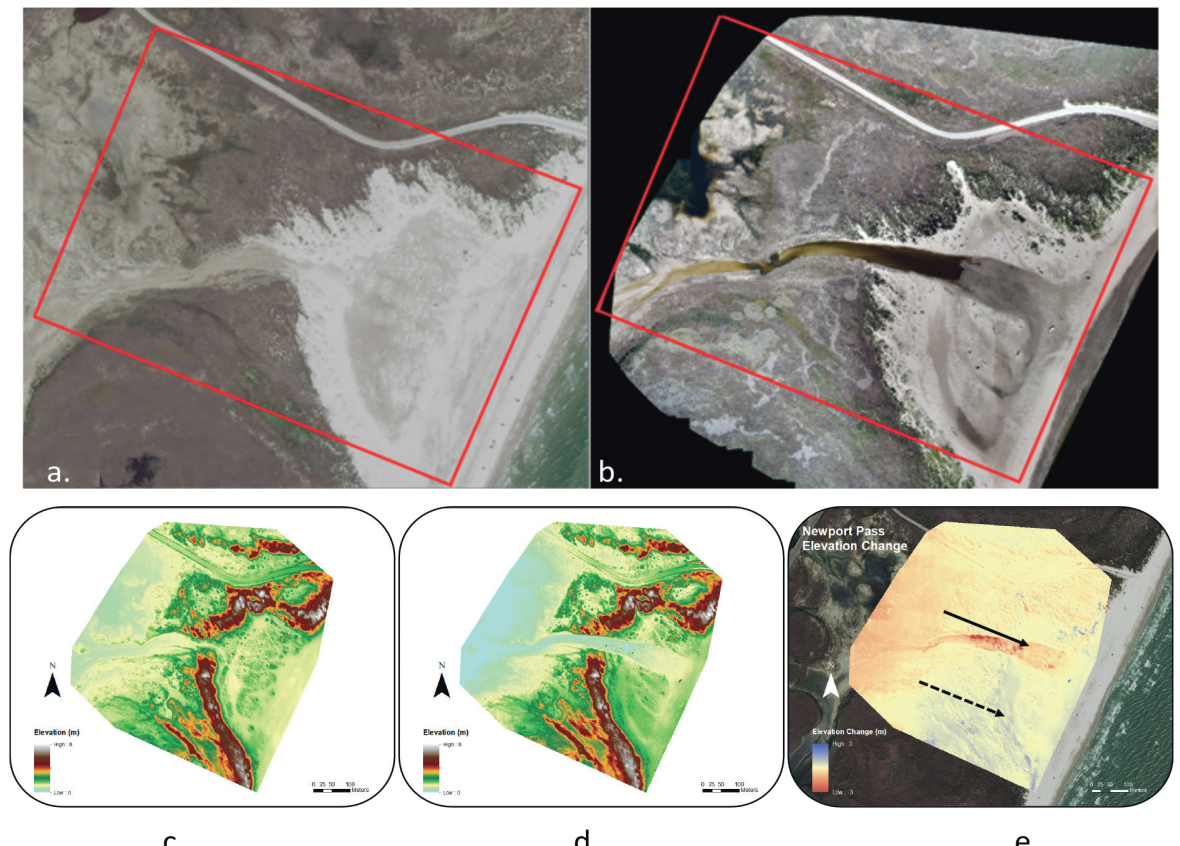

c.

d.

e.

\section{FIGURE 7.9}

These images depict storm-related changes to the Newport Pass study site. (a) Aerial image south of Newport Pass before Hurricane Harvey; (b) UAS imagery of Newport Pass after Hurricane Harvey; (c) DEM of Newport Pass created from a 2016 USACE airborne lidar survey before Hurricane Harvey; (d) DEM of Newport Pass created from a UAS survey after Hurricane Harvey; (e) Computed elevation change of Newport Pass due to Hurricane Harvey (before DEM surface subtracted from after DEM surface). Solid arrow shows zone of large erosion stemming from the breach. Dashed arrow shows zone of deposition.

\subsection{Conclusion}

Engineering and scientific solutions for sustainable development of cities and communities requires updated geospatial information. For communities residing within the highly dynamic and vulnerable coastal zone, accurate and timely geospatial data is critical to aid disaster response, mitigate risks to coastal hazards, and plan for more sustainable and resilient infrastructure. UAS is transforming our ability to map and monitor our evolving world at unprecedented detail. As UAS technology continues to rapidly evolve, so will the speed at which we can attain increasingly accurate and detailed spatial 


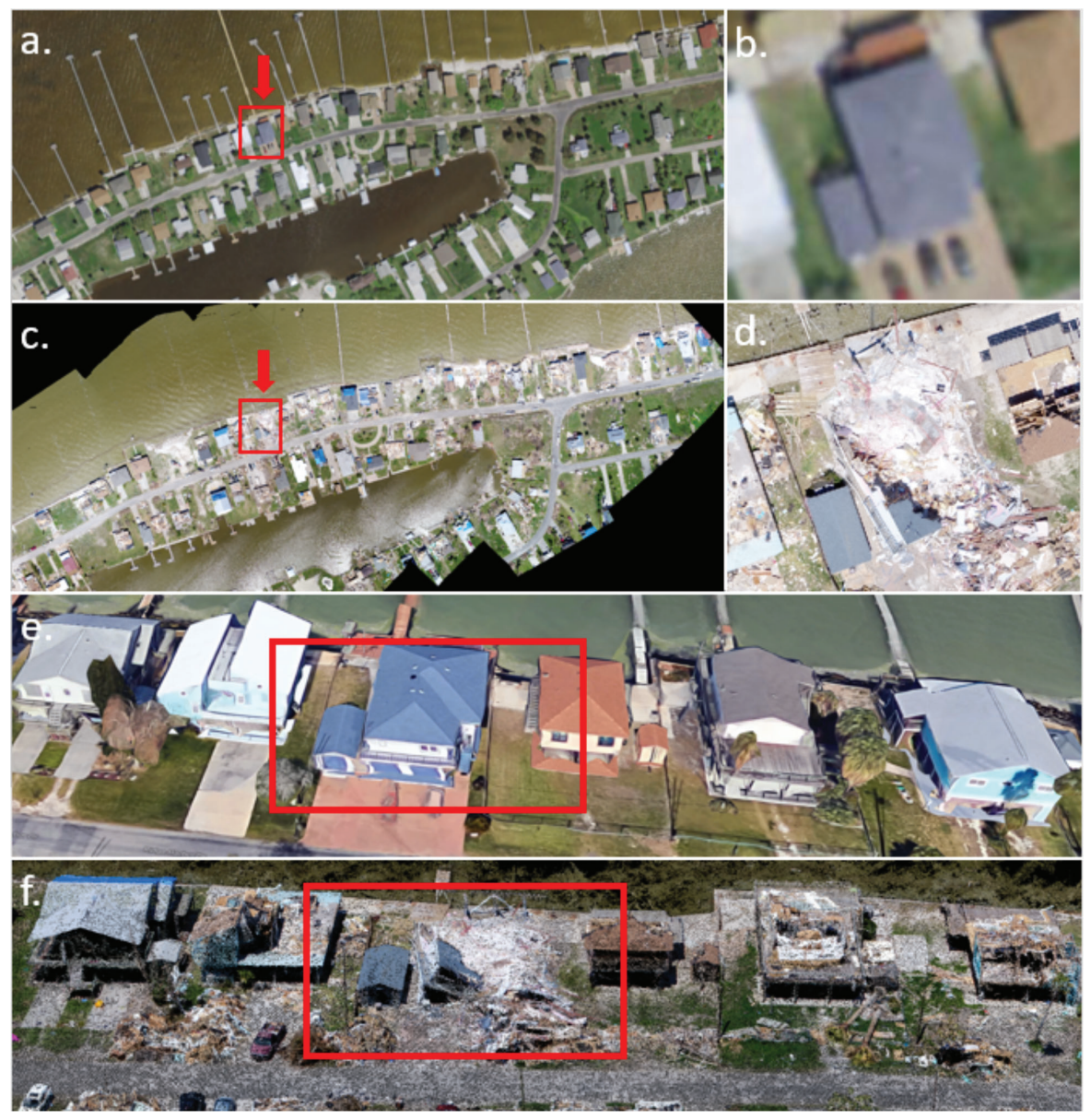

\section{FIGURE 7.10}

These images depict storm-related changes to a neighborhood near Rockport, TX: (a) Traditional aerial imagery of a Rockport neighborhood before Hurricane Harvey from Google Earth; (b) Zoomed in view of the aerial image showing the house before Hurricane Harvey; (c) UAS imagery of a Rockport neighborhood after Hurricane Harvey; (d) Zoomed in view of UAS imagery showing damage to the same house after Hurricane Harvey shown in the aerial image above; e) oblique imagery of a Rockport house before hurricane Harvey; (f) oblique view of a SfM derived 3D point cloud of the same house after hurricane Harvey.

information. With increasing reliance upon geospatial technology and data to inform our decisions, it becomes ever more important to understand the applications and limitations with such measurements and how to effectively apply 
them to better navigate our future world. It becomes ever more important to understand the accuracies associated with such measurements and how to effectively apply them to better navigate our future world.

\section{Bibliography}

[1] Designing the image acquisition plan, article 202557459, May 2018.

[2] United nations atlas of the oceans, October 2018.

[3] Jonathan L Carrivick, Mark W Smith, and Duncan J Quincey. Structure from Motion in the Geosciences. John Wiley \& Sons, 2016.

[4] Ismael Colomina and Pere Molina. Unmanned aerial systems for photogrammetry and remote sensing: A review. ISPRS Journal of photogrammetry and remote sensing, 92:79-97, 2014.

[5] National Research Council et al. Disaster resilience: A national imperative, 2012.

[6] F Greenwood. How to make maps with drones. Drones and aerial observation, July, pages 355-47, 2015.

[7] Richard JT Klein, Marion J Smit, Hasse Goosen, and Cornelis H Hulsbergen. Resilience and vulnerability: coastal dynamics or dutch dikes? Geographical Journal, pages 259-268, 1998.

[8] Richard Slocum and Christopher Parrish. Simulated imagery rendering workflow for uas-based photogrammetric $3 \mathrm{~d}$ reconstruction accuracy assessments. Remote Sensing, 9(4):396, 2017.

[9] Michael J Starek, Thomas Davis, Dan Prouty, and Jacob Berryhill. Smallscale uas for geoinformatics applications on an island campus. In 2014 Ubiquitous Positioning Indoor Navigation and Location Based Service (UPINLBS), pages 120-127. IEEE, 2014.

[10] Darren Turner, Arko Lucieer, and Christopher Watson. An automated technique for generating georectified mosaics from ultra-high resolution unmanned aerial vehicle (uav) imagery, based on structure from motion (sfm) point clouds. Remote sensing, 4(5):1392-1410, 2012.

[11] Geert Jan Van Oldenborgh, Karin Van Der Wiel, Antonia Sebastian, Roop Singh, Julie Arrighi, Friederike Otto, Karsten Haustein, Sihan Li, Gabriel Vecchi, and Heidi Cullen. Attribution of extreme rainfall from hurricane harvey, august 2017. Environmental Research Letters, 12(12):124009, 2017. 
[12] AS Woodget, PE Carbonneau, Fleur Visser, and IP Maddock. Quantifying submerged fluvial topography using hyperspatial resolution uas imagery and structure from motion photogrammetry. Earth Surface Processes and Landforms, 40(1):47-64, 2015. 